DOI: https://doi.org/10.24297/jam.v17i0.8443

\title{
Fuzzy Graphs
}

\author{
Huda Mutab Al Mutab \\ Computer Science Department, College of Science and Human studies in Durma, Shaqra University, Saudi Arabia \\ e-mail: halmutab@su.edu.sa
}

\begin{abstract}
In this paper, neighbourly irregular fuzzy graphs, neighbourly total irregular fuzzy graphs, highly irregular fuzzy graphs and highly total irregular fuzzy graphs are introduced. A necessary and sufficient condition under which neighbourly irregular and highly irregular fuzzy graphs are equivalent is provided. We define $d_{2}$ degree of a vertex in fuzzy graphs and total $d_{2}$-degree of a vertex in fuzzy graphs and $(2, k)$-regular fuzzy graphs, totally $(2, k)$ - regular fuzzy graphs are introduced. $(2, k)$ - regular fuzzy graphs and totally $(2, k)$-regular fuzzy graphs are compared through various examples.
\end{abstract}

Keywords: Fuzzy graph, Irregular fuzzy graph, regular fuzzy graph.

\section{Introduction}

In 1975, Rosenfeld considered fuzzy relations on fuzzy sets. He developed the theory of fuzzy graphs. Bang and Yeh during the same time introduced various connectedness concepts in fuzzy graph. Inexact information is used in expressing or describing human behaviors and mental process. The information depends upon a person subjectively and it is difficult to process objectively. Fuzzy information can be analyzed by using a fuzzy graph. Fuzzy graph is an expression of fuzzy relation and thus the fuzzy graph is frequently expressed in fuzzy matrix. N. R. Santhimaheswari and C. Sekar in 2016 On strongly edge irregular fuzzy graph. O. T. Manjusha, M. S. Sunitha,in (2016), Connected domination in fuzzy graphs. K. R. Sandeep Narayan and M. S. Sunitha in 2012, Connectivity in a fuzzy Graph and its complement. In 1965, Lotfi A. Zadeh introduced the concept of a fuzzy subset of a set as a method for representing the phenomena of uncertainty in real life situations. Azriel Rosenfeld introduced fuzzy graph in 1975. Alison Northup introduced semiregular graph also called as $(2, k)$-regular graph and studied some properties of $(2, k)$-regular graph. N. R. Santhi Maheswari and C. Sekar introduced $d_{2}$ of a vertex in graph and also discussed some properties on $d_{2}$ of a vertex in graph and introduced $(r, 2, k)$-regular graph and also discussed some properties of $(r, 2, k)$-regular graph. In this paper, we define $d_{2}$ degree of a vertex in fuzzy graphs and total $d_{2}$-degree of a vertex in fuzzy graphs and $(2, k)$-regular fuzzy graphs, totally $(2, k)$ - regular fuzzy graphs are introduced. $(2, k)$-regular fuzzy graphs and totally $(2, k)$-regular fuzzy graphs are compared through various examples.

\section{Graph}

Definition 1.1 (Crisp graph) A graph $G=(V, E)$ consists of $V$, a nonempty set of vertices (or nodes) and $E$, a set of edges. Each edge has either one or two vertices associated with it, called its endpoints. An edge is said to connect its endpoints.

ftbpFU3.5674in1.337in0ptExamples of crisp graphs 1Figure(1)

Definition 1.2 The degree of a vertex in an undirected graph is the number of edges incident with it, except that a loop at a vertex contributes twice to the degree of that vertex. The degree of the vertexv is denoted by deg $(v)$. 
Example 1.1 Figure (2) the degree of the vertex $b$ in the graph $H$ is deg $(b)=6$, because $b$ has loop at vertex.

Definition 1.3 The degree $d_{G}(v)$ of a vertex $v$ in $G$ or simply $d(v)$ is the number of edges of $G$ incident with vertex $v$.

ftbpFU5.8505in1.8922in0ptThe degree of a vertex in two undirected graphs $G$ and $H 2$ Figure(2)

Example 1.2 To find the degrees of the vertices in the graphs $G$ and $H$ displayed in Figure (2).

- In $G, \operatorname{deg}(a)=2, \operatorname{deg}(b)=\operatorname{deg}(c)=\operatorname{deg}(f)=4, \operatorname{deg}(d)=1, \operatorname{deg}(e)=3$,

and $\operatorname{deg}(g)=0$.

- In $H, \operatorname{deg}(a)=4, \operatorname{deg}(b)=\operatorname{deg}(e)=6, \operatorname{deg}(c)=1$, and $\operatorname{deg}(d)=5$.

Definition 1.4 A complete graph on $n$ vertices, denoted by $K_{n}$, is a simple graph that contains exactly one edge between each pair of distinct vertices. The graphs $K_{n}$, for $n=1,2,3,4,5,6$, are displayed in Figure (3). A simple graph for which there is at least one pair of distinct vertex not connected by an edge is called noncomplete.

Example 1.3 Figure (3) examples of Complete Graphs.

ftbpFU5.7735in2.0877in0ptComplete Graphs3Figure(3)

Definition 1.5 Cycles $A$ cycle $C_{n}, n \geq 3$, consists of $n$ vertices $v_{1}, v_{2}, \ldots, v_{n}$ and edges $\left\{v_{1}, v_{2}\right\},\left\{v_{1}, v_{2}\right\}, .$. . , $\left\{v_{n-1}, v_{n}\right\}$, and $\left\{v_{n}, v_{1}\right\}$. The cycles $C_{3}, C_{4}, C_{5}$, and $C_{6}$ are displayed in Figure (4).

ftbpFU4.7193in1.4105in0ptCycle of crisp graphs4Figure(4)

Definition 1.6 Let $n$ be a nonnegative integer and $G$ an undirected graph. A path of length $n$ from $u$ to $v$ in $G$ is a sequence of $n$ edges $e_{1}, \ldots, e_{n}$ of $G$ for which there exists a sequence $x_{0}=u, x_{1}, \ldots, x_{n-1}, x_{n}=v$ of vertices such that $e_{i}$ has, for $i=1, \ldots, n$, the endpoints $x_{i-1}$ and $x_{i}$. When the graph is simple, we denote this path by its vertex sequence $x_{0}, x_{1}, \ldots, x_{n}$ (because listing these vertices uniquely determines the path). The path is a circuit if it begins and ends at the same vertex, that is, if $u=v$, and has length greater than zero. The path or circuit is said to pass through the vertices $x_{1}, x_{2}, \ldots, x_{n-1}$ or traverse the edges $e_{1}, e_{2}, \ldots, e_{n}$. A path or circuit is simple if it does not contain the same edge more than once.

\section{ftbpFU2.3947in1.5575in0ptPath of graph5Figure(5)}

Definition 1.7 An undirected graph is called connected if there is a path between every pair of distinct vertices of the graph. An undirected graph that is not connected is called disconnected. We say that we disconnect a graph when we remove vertices or edges, or both, to produce a disconnected subgraph.

Example 1.4 The graph $G_{1}$ in Figure (6) is connected, because for every pair of distinct vertices there is a path between them. However, the graph $G_{2}$ in Figure (6) is not connected. For instance, there is no path in $G_{2}$ between vertices $a$ and $d$.

ftbpFU3.2015in2.1845in0ptConnected graphs and disconnected3.6Figure(3.6)

Definition 1.8 A subgraph of a graph $G=(V, E)$ is a graph $H=(W, F)$, where $W \subseteq V$ and $F \subseteq E$. A subgraph $H$ of $G$ is a proper subgraph of $G$ if $H \neq G$.

Example 1.5 Figure (7) examples of subgraphs of the original graph. 
ftbpFU3.4108in1.6302in0ptSubgraphs of the original graph3.7Figure(3.7)

Definition 1.9 The degree of an edge $(u, v)$ in the underlying graph is defined as $d_{G}(u, v)=d_{G}(u)+d_{G}(v)-2$.

Example 1.6 In figure (6) the degree of edge $(a, b)$ in graph $G, d_{G}(a, b)=d_{G}(a)+d_{G}(b)-2=2+2-2=2$.

Definition 1.10 A graph $G$ is called regular if every vertex is adjacent only to vertices having the same degree. A graph $G$ is called irregular, if there is a vertex which is adjacent only to vertices with distinct degrees.

Example 1.7 In figure (6) the graph $G_{1}$ is irregular graph, because

$d_{G}(a)=2, d_{G}(d)=3$. In figure $(7)$ he original graph is regular fuzzy graph, because every vertex is adjacent only to vertices having the same degree.

\section{Fuzzy graph}

Definition 2.1 A Fuzzy graph denoted by $\widetilde{G}=(V, E)$ on the graph $G=(V, E)$ is a pair of functions $(\sigma, \mu)$ where $\sigma: V \longrightarrow[0,1]$ is a fuzzy subset of a non empty set $V$ and $\mu: V \times V \longrightarrow[0,1]$ is a symmetric fuzzy relation on $\sigma$ such that for all $u$ and $v$ in $V$ the relation $\mu(u, v)=\mu(u v) \leq \sigma(u) \wedge \sigma(v)$ is satisfied.

Definition 2.2 A Fuzzy graph $\widetilde{G}=(\sigma, \mu)$ is a pair of functions $\sigma: V \longrightarrow[0,1]$ where $V$ is the set of vertices and $\mu$ $: V \times V \longrightarrow[0,1], \forall x, y \in V$. Fuzzy graph $\widetilde{H}=\left(\sigma_{1}, \mu_{1}\right)$ is called a fuzzy subgraph of $\widetilde{G}$ induced by $\left\langle V_{1}\right\rangle$ and $V_{1} \subseteq V$ if define $\sigma_{1}$ on $V_{1}, \sigma_{1}: V_{1} \longrightarrow[0,1], \sigma_{1}(x) \leq \sigma(x), \forall x \in V_{1}$ and $\mu_{1}$ on the collection $E_{1}$ of two element subsets of $V_{1}$ by

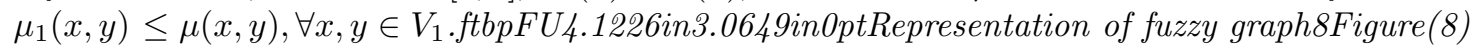

Example 2.1 Let $E=\left\{x_{1}, x_{2}, x_{3}, x_{4}, x_{5}\right\}$, then a fuzzy graph could be described as

$$
\begin{aligned}
& \widetilde{G}\left(x_{i}, x_{j}\right)=\left\{\left[\left(x_{1}, x_{2}\right), 0.3\right],\left[\left(x_{1}, x_{3}\right), 0.8\right],\left[\left(x_{1}, x_{1}\right), 0.7\right],\left[\left(x_{2}, x_{1}\right), 0.5\right]\right. \\
& ,\left[\left(x_{3}, x_{1}\right) 0.2\right],\left[\left(x_{3}, x_{2}\right), 0.6\right],\left[\left(x_{4}, x_{3}\right), 0.8\right],\left[\left(x_{1}, x_{5}\right), 0.9\right], \\
& \left.\left[\left(x_{3}, x_{5}\right), 0.2\right],\left[\left(x_{5}, x_{2}\right), 0.1\right]\right\} \text {. Then the fuzzy subgraph } \widetilde{H} \text { of } \widetilde{G} \text { is } \\
& \widetilde{H}\left(x_{i}, x_{j}\right)=\left\{\left[\left(x_{1}, x_{2}\right), 0.1\right],\left[\left(x_{1}, x_{3}\right), 0.4\right],\left[\left(x_{1}, x_{1}\right), 0.3\right],\left[\left(x_{3}, x_{1}\right) 0.1\right],\right. \\
& \left.\left[\left(x_{3}, x_{2}\right), 0.2\right],\left[\left(x_{4}, x_{3}\right), 0.7\right],\left[\left(x_{1}, x_{5}\right), 0.5\right],\left[\left(x_{3}, x_{5}\right), 0.2\right],\left[\left(x_{5}, x_{2}\right), 0.1\right]\right\} .
\end{aligned}
$$

Definition 2.3 Let $\widetilde{G}=(\sigma, \mu)$ be a fuzzy graph on $G=(V, E)$. The degree of a vertex $u$ is $d_{\widetilde{G}}(u)=\sum_{u \neq v \in E} \mu(u, v)$, for $(u, v) \in E$ and $\mu(u, v)=0$, for $(u, v)$ not in $E$, this is equivalent to $d_{\widetilde{G}}(u)=\sum_{(u, v) \in E} \mu(u, v)$.

Example 2.2 In fuzzy graph $\widetilde{G}$ above in figure $(8), d_{\widetilde{G}}\left(a_{1}\right)=1.0+0.5=1.5, d_{\widetilde{G}}\left(a_{2}\right)=1.0, d_{\widetilde{G}}\left(a_{3}\right)=1.0+0.5=1.5$, $d_{\widetilde{G}}\left(b_{1}\right)=1.0+0.5=1.5, d_{\widetilde{G}}\left(b_{2}\right)=1.0+0.5=1.5, d_{\widetilde{G}}\left(b_{3}\right)=1.0$.

Definition 2.4 Let $\widetilde{G}=(\sigma, \mu)$ be a fuzzy graph on $G=(V, E)$. The total degree of a vertex u is defined as

$$
t d_{\widetilde{G}}(u)=\sum \mu(u, v)+\sigma(u)=d_{\widetilde{G}}(u)+\sigma(u),(u, v) \in E .
$$

Example 2.3 Figure (9) Define $\widetilde{G}=(\sigma, \mu)$ by $\sigma(u)=0.9, \sigma(v)=0.8, \sigma(w)=0.7, \sigma(x)=0.6$, and $\mu(u, v)=0.2$, $\mu(u, w)=0.4, \mu(w, x)=0.4, \mu(x, v)=0.3$, then

$$
\begin{aligned}
& t d_{\widetilde{G}}(u)=\sum_{u \neq v} \mu(u, v)+\sigma(u)=d_{\widetilde{G}}(u)+\sigma(u)=0.7+0.9=1.6, t d_{\widetilde{G}}(v)=\sum_{u \neq v} \mu(u, v)+\sigma(v)=d_{\widetilde{G}}(v)+\sigma(v)= \\
& 0.5+0.8=1.3, t d_{\widetilde{G}}(w)=\sum_{w \neq u} \mu(u, w)+\sigma(w)=d_{\widetilde{G}}(w)+\sigma(w)=0.9+0.7=1.6, t d_{\widetilde{G}}(x)=\sum_{x \neq u} \mu(u, x)+\sigma(x)= \\
& d_{\widetilde{G}}(x)+\sigma(x)=0.7+0.6=0.3 .
\end{aligned}
$$


Definition 2.5 Let $\widetilde{G}=(\sigma, \mu)$ be a fuzzy graph on $G=(V, E)$. Then $\widetilde{G}$ is said to be a irregular fuzzy graph if there exists a vertex which is adjacent to a vertices with distinct degrees.

Example 2.4 Define $\widetilde{G}=(\sigma, \mu)$ by $\sigma(u)=0.4, \sigma(v)=0.6, \sigma(w)=0.4, \sigma(x)=0.2, \sigma(y)=0.5$, and $\mu(u, v)=0.2$, $\mu(v, w)=0.4, \mu(w, x)=0.3, \mu(x, y)=0.2, \mu(u, y)=0.3, \exists u, v \in \widetilde{G}$ such that $d_{\widetilde{G}}(u)=0.2+0.3=0.5$ and $d_{\widetilde{G}}(v)=0.2+0.4=0.6$, such that $u, v$ are adjacent vertices but $d_{\widetilde{G}}(u) \neq d_{\widetilde{G}}(v)$.

Definition 2.6 Let $\widetilde{G}=(\sigma, \mu)$ be a fuzzy graph on $G=(V, E)$. Then $\widetilde{G}$ is said to be a totally irregular fuzzy graph if there exists a vertex which is adjacent to a vertices with distinct total degrees.

ftbpFU2.6878in2.3627in0ptTotally irregular fuzzy graph9Figure(9)

Example 2.5 See Figure (9). Define $\widetilde{G}=(\sigma, \mu)$ by $\sigma(u)=0.9, \sigma(v)=0.8, \sigma(w)=0.7, \sigma(x)=0.6$, and $\mu(u, v)=0.2$, $\mu(u, w)=0.4, \mu(w, x)=0.4$,

$$
\begin{aligned}
\mu(x, v) & =0.3, \text { then } \\
t d_{\widetilde{G}}(u) & =\sum_{u \neq v} \mu(u, v)+\sigma(u)=d_{\widetilde{G}}(u)+\sigma(u)=0.7+0.9=1.6, \\
t d_{\widetilde{G}}(v) & =\sum_{u \neq v} \mu(u, v)+\sigma(v)=d_{\widetilde{G}}(v)+\sigma(v)=0.5+0.8=1.3, \\
t d_{\widetilde{G}}(w) & =\sum_{w \neq u} \mu(u, w)+\sigma(w)=d_{\widetilde{G}}(w)+\sigma(w)=0.9+0.7=1.6, \\
t d_{\widetilde{G}}(x) & =\sum_{x \neq u} \mu(u, v)+\sigma(x)=d_{\widetilde{G}}(x)+\sigma(x)=0.7+0.6=1.3 .
\end{aligned}
$$

$\exists u, v \in \widetilde{G}$ such that $t d_{\widetilde{G}}(u)=1.6, t d_{\widetilde{G}}(v)=1.3$, and $u, v$ are adjacent vertices, $t d_{\widetilde{G}}(u) \neq t d_{\widetilde{G}}(v)$.

$\widetilde{G}$ is a totally irregular fuzzy graph and an irregular fuzzy graph because, $\exists u, v \in \widetilde{G}$ such that $d_{\widetilde{G}}(u)=0.7, d_{\widetilde{G}}(v)=0.5$ and $u, v$ are adjacent vertices, $d_{\widetilde{G}}(u) \neq d_{\widetilde{G}}(v)$.

Definition 2.7 Let $\widetilde{G}=(\sigma, \mu)$ be a fuzzy graph on $G=(V, E)$. Then $\widetilde{G}$ is said to be a strongly irregular fuzzy graph if every pair of vertices have distinct degrees.

Example 2.6 Let $\widetilde{G}=(\sigma, \mu)$ be a fuzzy graph on $G=(V, E)$ which is a cycle of length five. From Figure (3.10), $d_{\widetilde{G}}(u)=0.6, d_{\widetilde{G}}(v)=0.3, d_{\widetilde{G}}(w)=0.5, d_{\widetilde{G}}(x)=0.7, d_{\widetilde{G}}(y)=0.9$. Every pair of vertices have distinct degrees.

Define $\widetilde{G}=(\sigma, \mu)$ by $\sigma(u)=0.4, \sigma(v)=0.3, \sigma(w)=0.5, \sigma(x)=0.7$, and $\mu(u, v)=0.3, \mu(v, w)=0.2$, $\mu(w, x)=0.3, \mu(x, u)=0.4 . \quad d_{\widetilde{G}}(u)=0.3+0.4=0.7, d_{\widetilde{G}}(v)=0.3+0.2=0.5, d_{\widetilde{G}}(w)=0.2+0.3=0.5$, $d_{\widetilde{G}}(x)=0.3+0.4=0.7, \widetilde{G}$ has two pair $\{v, w\},\{u, x\}$ vertices having the same degree then $\widetilde{G}$ is not strongly irregular fuzzy graph.

Definition 2.8 Let $\widetilde{G}=(\sigma, \mu)$ be a fuzzy graph on $G=(V, E)$. Then $\widetilde{G}$ is said to be a highly irregular fuzzy graph if every vertex in $\widetilde{G}$ is adjacent to the vertices having distinct degrees.

Example 2.7 Define $\widetilde{G}=(\sigma, \mu)$ by $\sigma(u)=0.4, \sigma(v)=0.3, \sigma(w)=0.5, \sigma(x)=0.7$, and $\mu(u, v)=0.3, \mu(v, w)=0.2$, $\mu(w, x)=0.3, \mu(x, u)=0.4 . \quad d_{\widetilde{G}}(u)=0.3+0.4=0.7, d_{\widetilde{G}}(v)=0.3+0.2=0.5, d_{\widetilde{G}}(w)=0.2+0.3=0.5$, $d_{\widetilde{G}}(x)=0.3+0.4=0.7$, every vertex in $\widetilde{G}$ is adjacent to the vertices having distinct degrees. then $\widetilde{G}$ is highly irregular fuzzy graph. 
Definition 2.9 Let $\widetilde{G}=(\sigma, \mu)$ be a fuzzy graph on $G=(V, E)$. Then $\widetilde{G}$ is said to be a neighbourly irregular fuzzy graph If every two adjacent vertices of a fuzzy graph $\widetilde{G}$ have distinct degree.

Example 2.8 Define $\widetilde{G}=(\sigma, \mu)$ by $\sigma(u)=0.8, \sigma(v)=0.9, \sigma(w)=0.7, \sigma(x)=0.6$, and $\mu(u, v)=0.8, \mu(v, w)=0.4$, $\mu(w, x)=0.6, \mu(x, u)=0.6 . \quad d_{\widetilde{G}}(u)=0.8+0.6=1.4, d_{\widetilde{G}}(v)=0.4+0.8=1.2, d_{\widetilde{G}}(w)=0.4+0.6=1.0$, $d_{\widetilde{G}}(x)=0.6+0.6=1.2$. We note that every two adjacent vertices of a fuzzy graph $\widetilde{G}$ have distinct degree. Then $\widetilde{G}$ neighbourly irregular fuzzy graph.

Remark 2.1 A highly irregular fuzzy graph need not be a neighbourly irregular fuzzy graph.

Example 2.9 Define $\widetilde{G}=(\sigma, \mu)$ by $\sigma(u)=0.7, \sigma(v)=0.8, \sigma(x)=0.5, \sigma(y)=0.9, \sigma(z)=0.6$, and $\mu(u, v)=0.4$, $\mu(v, x)=0.4, \mu(x, y)=0.5, \mu(u, y)=0.4, \mu(y, z)=0.6 . \quad d_{\widetilde{G}}(u)=0.4+0.4=0.8, d_{\widetilde{G}}(v)=0.4+0.4=0.8$, $d_{\widetilde{G}}(x)=0.4+0.5=0.9, d_{\widetilde{G}}(y)=0.5+0.4+0.6=1.5, d_{\widetilde{G}}(z)=0.6$.

To every vertex $v \in V$, the adjacent vertices have distinct degrees.

Hence $\widetilde{G}$ is highly irregular. But $d_{\widetilde{G}}(u)=d_{\widetilde{G}}(v)=0.8$.

So $\widetilde{G}$ is not neighbourly irregular.

Remark 2.2 A neighbourly irregular fuzzy graph need not be a highly irregular fuzzy graph.

Example 2.10 Define $\widetilde{G}=(\sigma, \mu)$ by $\sigma(u)=0.6, \sigma(v)=0.4, \sigma(w)=0.5, \sigma(y)=0.5, \sigma(x)=0.7$, and $\mu(u, v)=0.6$, $\mu(v, w)=0.3, \mu(w, y)=0.2, \mu(u, y)=0.4, \mu(w, x)=0.5, \mu(x, y)=0.3 . d_{\widetilde{G}}(u)=0.6+0.4=1.0, d_{\widetilde{G}}(v)=0.6+0.3=$ $0.9, d_{\widetilde{G}}(w)=0.3+0.2=0.5, d_{\widetilde{G}}(y)=0.2+0.4+0.3=0.9, d_{\widetilde{G}}(x)=0.5+0.3=0.8$. No two adjacent vertices has the same degree. So $\widetilde{G}$ is a neighbourly irregular fuzzy graph. But to the vertex $u$, the adjacent vertices $v$ and $y$ has the same degree $(=0.9)$ such that $d_{\widetilde{G}}(u)=1.0, d_{\widetilde{G}}(v)=0.9, d_{\widetilde{G}}(y)=0.9$. So $\widetilde{G}$ is not highly irregular fuzzy graph.

Definition 2.10 $\widetilde{G}=(\sigma, \mu)$ be a fuzzy graph. The degree of an edge $(u, v)$ is defined as $d_{\widetilde{G}}(u, v)=d_{\widetilde{G}}(u)+d_{\widetilde{G}}(v)$ $-2 \mu(u, v)$. The minimum degree of an edge is $\delta_{E}(\widetilde{G})=\wedge\left\{d_{\widetilde{G}}(u, v):(u, v) \in E\right\}$. The maximum degree of an edge is $\Delta_{E}(\widetilde{G})=\vee\left\{d_{\widetilde{G}}(u, v):(u, v) \in E\right\}$.

Example 2.11 In the above example (3.10). Define $\widetilde{G}=(\sigma, \mu)$ by $\sigma(u)=0.6$,

$$
\begin{aligned}
& \sigma(v)=0.4, \sigma(w)=0.5, \sigma(y)=0.5, \sigma(x)=0.7 \\
& \text { and } \mu(u, v)=0.6, \mu(v, w)=0.3, \mu(w, y)=0.2, \\
& \mu(u, y)=0.4, \mu(w, x)=0.5, \mu(x, y)=0.3 . \\
& d_{\widetilde{G}}(u)=0.6+0.4=1.0, d_{\widetilde{G}}(v)=0.6+0.3=0.9, \\
& d_{\widetilde{G}}(w)=0.3+0.2=0.5, d_{\widetilde{G}}(y)=0.2+0.4+0.3=0.9, \\
& d_{\widetilde{G}}(x)=0.5+0.3=0.8 . \text { Then } \\
& d_{\widetilde{G}}(u, v)=d_{\widetilde{G}}(u)+d_{\widetilde{G}}(v)-2 \mu(u, v)=1.0+0.9-2(0.6)=0.7, \\
& d_{\widetilde{G}}(v, w)=d_{\widetilde{G}}(v)+d_{\widetilde{G}}(w)-2 \mu(v, w)=0.9+0.5-2(0.3)=0.8, \\
& d_{\widetilde{G}}(w, y)=d_{\widetilde{G}}(w)+d_{\widetilde{G}}(y)-2 \mu(w, y)=0.5+0.6-2(0.2)=0.7, \\
& d_{\widetilde{G}}(u, y)=d_{\widetilde{G}}(u)+d_{\widetilde{G}}(y)-2 \mu(u, y)=1.0+0.6-2(0.4)=0.8, \\
& d_{\widetilde{G}}(w, x)=d_{\widetilde{G}}(w)+d_{\widetilde{G}}(x)-2 \mu(w, y)=0.5+0.8-2(0.5)=0.3, \\
& d_{\widetilde{G}}(x, y)=d_{\widetilde{G}}(x)+d_{\widetilde{G}}(y)-2 \mu(x, y)=0.8+0.6-2(0.3)=0.8, \\
& \delta_{E}(\widetilde{G})=\wedge\left\{d_{\widetilde{G}}(u, v):(u, v) \in E\right\}=\min \{0.7,0.8,0.7,0.8,0.3,0.8\}=0.3, \\
& \Delta_{E}(\widetilde{G})=\vee\left\{d_{\widetilde{G}}(u, v):(u, v) \in E\right\}=\max \{0.7,0.8,0.7,0.8,0.3,0.8\}=0.8 .
\end{aligned}
$$


Definition 2.11 Let $\widetilde{G}=(\sigma, \mu)$ be a fuzzy graph. The total degree of an edge $(u, v)$ is defined as td $\widetilde{G}(u, v)=$ $d_{\widetilde{G}}(u)+d_{\widetilde{G}}(v)-\mu(u, v)$. The minimum total degree of an edge is $\delta_{t E}(\widetilde{G})=\wedge\left\{t d_{\widetilde{G}}(u, v):(u, v) \in E\right\}$. The maximum total degree of an edge is $\Delta_{t E}(\widetilde{G})=\vee\left\{t d_{\widetilde{G}}(u, v):(u, v) \in E\right\}$.

Example 2.12 Define $\widetilde{G}=(\sigma, \mu)$ by $\sigma(u)=0.6, \sigma(v)=0.4, \sigma(w)=0.5, \sigma(y)=0.5, \sigma(x)=0.7$, and $\mu(u, v)=0.6$, $\mu(v, w)=0.3, \mu(w, y)=0.2, \mu(u, y)=0.4, \mu(w, x)=0.5, \mu(x, y)=0.3$.

$$
\begin{aligned}
& d_{\widetilde{G}}(u)=0.6+0.4=1.0, d_{\widetilde{G}}(v)=0.6+0.3=0.9, \\
& d_{\widetilde{G}}(w)=0.3+0.2=0.5, d_{\widetilde{G}}(y)=0.2+0.4+0.3=0.9, \\
& d_{\widetilde{G}}(x)=0.5+0.3=0.8 . \text { Then } \\
& t d_{\widetilde{G}}(u, v)=d_{\widetilde{G}}(u)+d_{\widetilde{G}}(v)-\mu(u, v)=1.0+0.9-0.6=1.3, \\
& t d_{\widetilde{G}}(v, w)=d_{\widetilde{G}}(v)+d_{\widetilde{G}}(w)-\mu(v, w)=0.9+0.5-0.3=1.1, \\
& t d_{\widetilde{G}}(w, y)=d_{\widetilde{G}}(w)+d_{\widetilde{G}}(y)-\mu(w, y)=0.5+0.6-0.2=0.9, \\
& t d_{\widetilde{G}}(u, y)=d_{\widetilde{G}}(u)+d_{\widetilde{G}}(y)-\mu(u, y)=1.0+0.6-0.4=1.2, \\
& t d_{\widetilde{G}}(w, x)=d_{\widetilde{G}}(w)+d_{\widetilde{G}}(x)-\mu(w, y)=0.5+0.8-0.5=0.8, \\
& t d_{\widetilde{G}}(x, y)=d_{\widetilde{G}}(x)+d_{\widetilde{G}}(y)-\mu(x, y)=0.8+0.6-0.3=1.1, \\
& \delta_{t E}(\widetilde{G})=\wedge\left\{t d_{\widetilde{G}}(u, v):(u, v) \in E\right\}=\min \{1.3,1.1,0.9,1.2,0.8,1.1\}=0.8, \\
& \Delta_{t E}(\widetilde{G})=\vee\left\{t d_{\widetilde{G}}(u, v):(u, v) \in E\right\}=\max \{1.3,1.1,0.9,1.2,0.8,1.1\}=1.3 .
\end{aligned}
$$

\section{Connected Fuzzy Graphs}

Definition 3.1 (A path of fuzzy graph) In a fuzzy graph $\widetilde{G}=(V, \sigma, \mu)$, a path $P$ of length $n$ is a sequence of distinct nodes $u_{0}, u_{1}, \ldots, u_{n}$ such that $\mu\left(u_{i-1}, u_{i}\right)>0 ; i=1,2, \ldots, n$ and the degree of membership of a weakest arc is defined as its strength. If $u_{0}=u_{n}$ and $n>3$ then $P$ is called a cycle and $P$ is called a fuzzy cycle, if it contains more than one weakest arc. The strength of a cycle is the strength of the weakest arc in it. The strength of connectedness between two nodes $x$ and $y$ is defined as the maximum of the strengths of all paths between $x$ and $y$ and is denoted by

$C O N N_{\widetilde{G}}(x, y)=\max \left\{\mu\left(x, u_{1}\right) \wedge \mu\left(u_{1}, u_{2}\right) \wedge \ldots . \wedge \mu\left(u_{n-1}, y\right) \mid x, u_{1}, \ldots, u_{n-1}, y \in V\right\}$

, then $x, y$ are connected by path of length $n$.

Example 3.1 In Figure (9) the fuzzy graph is connected fuzzy graph the path from $u$ to $w$ is $(u, v, x, w)$ of length 3 $C O N N_{\widetilde{G}}(u, w)=0.2>0$.

Definition 3.2 A fuzzy graph $\widetilde{G}(\sigma, \mu)$ is connected if for every $x, y$ in $V$,

$$
\mu_{\widetilde{G}}(x, y)=C O N N_{\widetilde{G}}(x, y)>0 .
$$

Example 3.2 The same in example (4.1) above see figure (9).

Definition 3.3 Let $\widetilde{G}=(\sigma, \mu)$ be a connected fuzzy graph on $G=(V, E)$. Then $G$ is said to be a neighbourly edge irregular fuzzy graph if every pair of adjacent edges having distinct degrees.

Example 3.3 In the same example (3.8). Define $\widetilde{G}=(\sigma, \mu)$ by $\sigma(u)=0.8$,

$$
\sigma(v)=0.9, \sigma(w)=0.7, \sigma(x)=0.6, \text { and }
$$




$$
\begin{aligned}
& \mu(u, v)=0.8, \mu(v, w)=0.4, \mu(w, x)=0.6, \mu(x, u)=0.6 . \\
& \text { Then } d_{\widetilde{G}}(u)=0.8+0.6=1.4, d_{\widetilde{G}}(v)=0.8+0.4=1.2, \\
& d_{\widetilde{G}}(w)=0.4+0.6=1.0, d_{\widetilde{G}}(x)=0.6+0.6=1.2, \\
& d_{\widetilde{G}}(u, v)=d_{\widetilde{G}}(u)+d_{\widetilde{G}}(v)-2 \mu(u, v)=1.4+1.2-2(0.8)=1, \\
& d_{\widetilde{G}}(v, w)=d_{\widetilde{G}}(v)+d_{\widetilde{G}}(w)-2 \mu(v, w)=1.2+1.0-2(0.4)=1.4, \\
& d_{\widetilde{G}}(w, x)=d_{\widetilde{G}}(w)+d_{\widetilde{G}}(x)-2 \mu(w, x)=1.0+1.2-2(0.6)=1, \\
& d_{\widetilde{G}}(x, u)=d_{\widetilde{G}}(x)+d_{\widetilde{G}}(u)-2 \mu(x, u)=1.2+1.4-2(0.6)=1.4 .
\end{aligned}
$$

Definition 3.4 Let $\widetilde{G}=(\sigma, \mu)$ be a connected fuzzy graph on $G=(V, E)$. Then $\widetilde{G}$ is said to be a neighbourly edge totally irregular fuzzy graph if every pair of adjacent edges having distinct total degrees.

Example 3.4 Let $\widetilde{G}=(\sigma, \mu)$ be a fuzzy graph on $G=(V, E)$ which is a cycle of length five. From Figure (10), $d_{\widetilde{G}}(u)=0.6, d_{\widetilde{G}}(v)=0.3, d_{\widetilde{G}}(w)=0.5, d_{\widetilde{G}}(x)=0.7, d_{\widetilde{G}}(y)=0.9$. Total degrees of the edges are calculated as follows:

$$
\begin{aligned}
& t d_{\widetilde{G}}(u, v)=d_{\widetilde{G}}(u, v)+\mu(u, v)=0.7+0.1=0.8, \\
& t d_{\widetilde{G}}(v, w)=d_{\widetilde{G}}(v, w)+\mu(v, w)=0.4+0.2=0.6, \\
& t d_{\widetilde{G}}(w, x)=d_{\widetilde{G}}(w, x)+\mu(w, x)=0.6+0.3=0.9, \\
& t d_{\widetilde{G}}(x, y)=d_{\widetilde{G}}(x, y)+\mu(x, y)=0.8+0.4=1.2, \\
& t d_{\widetilde{G}}(y, u)=d_{\widetilde{G}}(y, u)+\mu(y, u)=0.5+0.5=1 .
\end{aligned}
$$

We note that every pair of adjacent edges having distinct total degrees. Then $\widetilde{G}$ is neighbourly edge totally irregular fuzzy graph.

Definition 3.5 A fuzzy graph $\widetilde{G}=(\sigma, \mu)$ is said to be complete if $\mu(u, v)=\sigma(u) \wedge \sigma(v)$, for all $u, v \in V$ and is denoted by $K_{\sigma}$.

Example 3.5 Let $\widetilde{G}$ be fuzzy graph Define $\widetilde{G}=(\sigma, \mu)$ by $\sigma(u)=0.8$,

$$
\begin{aligned}
& \sigma(v)=0.9, \sigma(w)=0.7, \sigma(x)=0.6, \text { and } \\
& \mu(u, v)=0.8, \mu(v, w)=0.7, \mu(w, x)=0.6, \mu(x, v)=0.6 .
\end{aligned}
$$

Then $\widetilde{G}=(\sigma, \mu)$ is complete fuzzy graph.

Because $\mu(u, v)=\sigma(u) \wedge \sigma(v)$, for all $u, v \in V$.

Definition 3.6 The order $p$ and size $q$ of a fuzzy graph $G=(\sigma, \mu)$ are defined to be $p=\sum_{x \in V} \sigma(x)$, and $q=$ $\sum_{(x, y) \in V \times V} \mu(x, y)$.

Example 3.6 Define $\widetilde{G}=(\sigma, \mu)$ by $\sigma(u)=0.8, \sigma(v)=0.9, \sigma(w)=0.7, \sigma(x)=0.6$, and $\mu(u, v)=0.8, \mu(v, w)=0.4$, $\mu(w, x)=0.6$, the order of $\widetilde{G}$ :

$$
p=\sum_{x \in V} \sigma(x)=\sigma(u)+\sigma(v)+\sigma(w)+\sigma(x)=0.8+0.9+0.7+0.6=2.5
$$

The size of $\widetilde{G}$ :

$$
q=\sum_{(x, y) \in V \times V} \mu(x, y)=\mu(u, v)+\mu(v, w)+\mu(w, x)=0.8+0.4+0.6+0.6=2.4 .
$$


Definition 3.7 Let $\widetilde{G}=(\sigma, \mu)$ be a fuzzy graph and $S \subseteq V$. Then the scalar cardinality of $S$ is defined to be $\sum_{v \in S} \sigma(v)$ and it is denoted by $|S|$. Let $p$ denotes the scalar cardinality of $V$, also called the order of $\widetilde{G}$.

Example 3.7 Let $\widetilde{G}$ be fuzzy graph Define $\widetilde{G}=(\sigma, \mu)$ by $\sigma(u)=0.8, \sigma(v)=0.9, \sigma(w)=0.7, \sigma(x)=0.6$, and $\mu(u, v)=0.8, \mu(v, w)=0.7, \mu(w, x)=0.6, \mu(x, v)=0.6$. Let $S=\{u, v, x\} \subseteq V$, then the scalar cardinality of $S$ is $|S|=\sum_{v \in S} \sigma(v)=0.8+0.9+0.6=2.3$

Notation 1 A node $u$ is said to be isolated if $\mu(u, v)=0$ for all $v \neq u$.

Definition 3.8 Let $\widetilde{G}=(\sigma, \mu)$ be a connected fuzzy graph on $G=(V, E)$. Then $\widetilde{G}$ is said to be a strongly edge irregular fuzzy graph if every pair of edges having distinct degrees (or) no two edges have same degree.

Example 3.8 Let $\widetilde{G}=(\sigma, \mu)$ be a fuzzy graph on $G=(V, E)$ which is a cycle of length five. From Figure (10), $d_{\widetilde{G}}(u)=0.6, d_{\widetilde{G}}(v)=0.3, d_{\widetilde{G}}(w)=0.5, d_{\widetilde{G}}(x)=0.7, d_{\widetilde{G}}(y)=0.9$.

Degrees of the edges are calculated as follows:

$$
\begin{aligned}
& d_{\widetilde{G}}(u, v)=d_{\widetilde{G}}(u)+d_{\widetilde{G}}(v)-2 \mu(u, v)=0.6+0.3-2(0.1)=0.7, \\
& d_{\widetilde{G}}(v, w)=d_{\widetilde{G}}(v)+d_{\widetilde{G}}(w)-2 \mu(v, w)=0.3+0.5-2(0.2)=0.4, \\
& d_{\widetilde{G}}(w, x)=d_{\widetilde{G}}(w)+d_{\widetilde{G}}(x)-2 \mu(w, x)=0.5+0.7-2(0.3)=0.6, \\
& d_{\widetilde{G}}(x, y)=d_{\widetilde{G}}(x)+d_{\widetilde{G}}(y)-2 \mu(x, y)=0.7+0.9-2(0.4)=0.8, \\
& d_{\widetilde{G}}(y, u)=d_{\widetilde{G}}(y)+d_{\widetilde{G}}(u)-2 \mu(y, u)=0.9+0.6-2(0.5)=0.5 .
\end{aligned}
$$

Definition 3.9 Let $G=(\sigma, \mu)$ be a connected fuzzy graph on $G=(V, E)$. Then $\widetilde{G}$ is said to be a strongly edge totally irregular fuzzy graph if every pair of edges having distinct total degrees (or) no two edges have same total degree.

ftbpFU3.8078in2.6775in0ptStrongly edge irregular fuzzy graph10qFigure(10q)

We noted that every pair of edges having distinct degrees. Hence $\widetilde{G}$ is strongly edge irregular fuzzy graph.

Example 3.9 Let $\widetilde{G}=(\sigma, \mu)$ be a fuzzy graph on $G=(V, E)$ which is a cycle of length five. From Figure (10), $d_{\widetilde{G}}(u)=0.6, d_{\widetilde{G}}(v)=0.3, d_{\widetilde{G}}(w)=0.5, d_{\widetilde{G}}(x)=0.7, d_{\widetilde{G}}(y)=0.9$. Total degrees of the edges are calculated as follows:

$$
\begin{aligned}
& t d_{\widetilde{G}}(u, v)=d_{\widetilde{G}}(u, v)+\mu(u, v)=0.7+0.1=0.8, \\
& t d_{\widetilde{G}}(v, w)=d_{\widetilde{G}}(v, w)+\mu(v, w)=0.4+0.2=0.6, \\
& t d_{\widetilde{G}}(w, x)=d_{\widetilde{G}}(w, x)+\mu(w, x)=0.6+0.3=0.9, \\
& t d_{\widetilde{G}}(x, y)=d_{\widetilde{G}}(x, y)+\mu(x, y)=0.8+0.4=1.2, \\
& t d_{\widetilde{G}}(y, u)=d_{\widetilde{G}}(y, u)+\mu(y, u)=0.5+0.5=1 .
\end{aligned}
$$

It is noted that every pair of edges in $\widetilde{G}$ having distinct total degrees. So, $\widetilde{G}$ is strongly edge totally irregular fuzzy graph. Hence $\widetilde{G}$ is both strongly edge irregular fuzzy graph and strongly edge totally irregular fuzzy graph. 
Remark 3.1 Graph in Figure (10) is both strongly edge irregular fuzzy graph and strongly edge totally irregular fuzzy graph.ftbpFU2.898in2.1326in0ptStrongly edge but not strongly edge totally11Figure(11)

Example 3.10 Strongly edge irregular fuzzy graphs need not be strongly edge totally irregular fuzzy graphs.

From Figure $(11), d_{\widetilde{G}}(u)=0.5, d_{\widetilde{G}}(v)=0.7$,

$d_{\widetilde{G}}(w)=0.6$. Also, $d_{\widetilde{G}}(u, v)=0.6, d_{\widetilde{G}}(v, w)=0.5$,

$d_{\widetilde{G}}(w, u)=0.7, t d_{\widetilde{G}}(u, v)=0.9, t d_{\widetilde{G}}(v, w)=0.9, t d_{\widetilde{G}}(w, u)=0.9$.

We noted that $\widetilde{G}$ is strongly edge irregular fuzzy graph, since every pair of edges having distinct degrees. Also, $\widetilde{G}$ is not strongly edge totally irregular fuzzy graph, since all the edges having same total degree. Hence strongly edge irregular fuzzy graph need not be strongly edge totally irregular fuzzy graph.

ftbpFU4.0707in2.0384in0ptStrongly edge totally need not be strongly edge 12Figure(12)

Example 3.11 Strongly edge totally irregular fuzzy graph need not be strongly edge irregular fuzzy graph. Consider $G=(V, E)$ where $V=\{u ; v ; w ; x\}$ and $E=\{(u, v),(v, w),(w, x),(x, u)\}$. And $\widetilde{G}=(\sigma, \mu)$, be fuzzy graph From Figure (12) $d_{\widetilde{G}}(u)=1.1, d_{\widetilde{G}}(v)=0.8, d_{\widetilde{G}}(w)=1.2, d_{\widetilde{G}}(x)=1.5 . d_{\widetilde{G}}(u, v)=1.3, d_{\widetilde{G}}(v, w)=1, d_{\widetilde{G}}(w, x)=1.3, d_{\widetilde{G}}(x, u)=1$. It is noted that $d_{\widetilde{G}}(u, v)=d_{\widetilde{G}}(w, x)=1.3$. Hence $\widetilde{G}$ is not strongly edge irregular fuzzy graph. Also, $t d_{\widetilde{G}}(u, v)=1.6$, $t d_{\widetilde{G}}(v, w)=1.5, t d_{\widetilde{G}}(w, x)=2, t d_{\widetilde{G}}(u, v)=1.8$. It is observed that $t d_{\widetilde{G}}(u, v) \neq t d_{\widetilde{G}}(v, w) \neq t d_{\widetilde{G}}(w, x) \neq t d_{\widetilde{G}}(x, u)$. So, $\widetilde{G}$ is strongly edge totally irregular fuzzy graph. Hence strongly edge totally irregular fuzzy graph need not be strongly edge irregular fuzzy graph.

Theorem 3.1 Let $\widetilde{G}=(\sigma, \mu)$ be a connected fuzzy graph on $G=(V, E)$ and is constant function. If $\widetilde{G}$ is strongly edge irregular fuzzy graph, then $\widetilde{G}$ is strongly edge totally irregular fuzzy graph.

Assume that is a constant function, let $\mu(u, v)=c$ for all $(u, v) \in E$,

where $c$ is constant. Let $(u, v)$ and $(x, y)$ be any pair of edges in $E$.

Suppose that $\widetilde{G}$ is strongly edge irregular fuzzy graph.

Then $d_{\widetilde{G}}(u, v) \neq d_{\widetilde{G}}(x, y)$, where

$(u, v)$ and $(x, y)$ are any pair of edges in $E$.

$\Longrightarrow d_{\widetilde{G}}(u, v) \neq d_{\widetilde{G}}(x, y) \Longrightarrow d_{\widetilde{G}}(u, v)+c \neq d_{\widetilde{G}}(x, y)+c$

$\Longrightarrow d_{\widetilde{G}}(u, v)+\mu(u, v) \neq d_{\widetilde{G}}(x, y)+\mu(x, y)$

$\Longrightarrow t d_{\widetilde{G}}(u, v) \neq t d_{\widetilde{G}}(x, y)$,

where $(u, v)$ and $(x, y)$ are any pair of edges in $E$.

Hence $G$ is strongly edge total irregular fuzzy graph.

Theorem 3.2 Let $\widetilde{G}=(\sigma, \mu)$ be a connected fuzzy graph on $G=(V, E)$ and $\mu$ is constant function. If $\widetilde{G}$ is strongly edge totally irregular fuzzy graph, then $\widetilde{G}$ is strongly edge irregular fuzzy graph.

Assume that $\mu$ is a constant function, let $\mu(u, v)=c$ for all $(u, v) \in E$,

where $c$ is constant. Let $(u, v)$ and $(x, y)$ be any pair of edges in $E$.

Suppose that $\widetilde{G}$ is strongly edge totally irregular fuzzy graph.

Then $t d_{\widetilde{G}}(u, v) \neq t d_{\widetilde{G}}(x, y)$,

where $(u, v)$ and $(x, y)$ are any pair of edges in $E$.

$\Longrightarrow t d_{\widetilde{G}}(u, v) \neq t d_{\widetilde{G}}(x, y)$ 


$$
\begin{aligned}
& \Longrightarrow d_{\widetilde{G}}(u, v)+\mu(u, v) \neq d_{\widetilde{G}}(x, y)+\mu(x, y) \\
& \Longrightarrow d_{\widetilde{G}}(u, v)+c \neq d_{\widetilde{G}}(x, y)+c \\
& \Longrightarrow d_{\widetilde{G}}(u, v) \neq d_{\widetilde{G}}(x, y) .
\end{aligned}
$$

Where $(u, v)$ and $(x, y)$ are any pair of edges in $E$.

Hence $\widetilde{G}$ is strongly edge irregular fuzzy graph.

Remark 3.2 Let $\widetilde{G}=(\sigma, \mu)$ be a connected fuzzy graph on $G=(V, E)$. If $\widetilde{G}$ is both strongly edge irregular fuzzy graph and strongly edge totally irregular fuzzy graph. Then need not be a constant function.

ftbpFU3.8078in2.6775in0pt $\mu$ is not constant function13Figure(13)

Example 3.12 Let $\widetilde{G}=(\sigma, \mu)$ be a fuzzy graph on $G=(V, E)$ which is a cycle of length five. From Figure (13),

$d_{\widetilde{G}}(u)=0.6, d_{\widetilde{G}}(v)=0.3, d_{\widetilde{G}}(w)=0.5, d_{\widetilde{G}}(x)=0.7, d_{\widetilde{G}}(y)=0.9$. Also, $d_{\widetilde{G}}(u, v)=0.7, d_{\widetilde{G}}(v, w)=0.4, d_{\widetilde{G}}(w, x)$ $=0.6, d_{\widetilde{G}}(x, y)=0.8, d_{\widetilde{G}}(y, u)=0.5$. It is noted that every pair of edges in $\widetilde{G}$ having distinct degrees. Hence $\widetilde{G}$ is strongly edge irregular fuzzy graph. Also, $t d_{\widetilde{G}}(u, v)=0.8, t d_{\widetilde{G}}(v, w)=0.6, t d_{\widetilde{G}}(w, x)=0.9, t d_{\widetilde{G}}(x, y)=1.2$, $t d_{\widetilde{G}}(y, u)=1$. Note that every pair of edges in $\widetilde{G}$ having distinct total degrees. Hence $\widetilde{G}$ is both strongly edge irregular fuzzy graph and strongly edge totally irregular fuzzy graph. But $\mu$ is not constant function.

Theorem 3.3 Let $\widetilde{G}=(\sigma, \mu)$ be a fuzzy graph on $G=(V, E)$. If $\widetilde{G}$ is strongly edge irregular fuzzy graph, then $\widetilde{G}$ is neighbourly edge irregular fuzzy graph.

Let $\widetilde{G}=(\sigma, \mu)$ be a fuzzy graph on $G=(V, E)$. Let us assume that $\widetilde{G}$ is strongly edge irregular fuzzy graph then every pair of edges in $\widetilde{G}$ have distinct degrees so every pair of adjacent edges have distinct degrees. Hence $\widetilde{G}$ is neighbourly edge irregular fuzzy graph.

Theorem 3.4 Let $\widetilde{G}=(\sigma, \mu)$ be a fuzzy graph on $G=(V, E)$. If $\widetilde{G}$ is strongly edge totally irregular fuzzy graph, then $\widetilde{G}$ is neighbourly edge totally irregular fuzzy graph.

Let $\widetilde{G}=(\sigma, \mu)$ be a fuzzy graph on $G=(V, E)$. Let us assume that $\widetilde{G}$ is strongly edge totally irregular fuzzy graph then every pair of edges in $\widetilde{G}$ have distinct total degrees so every pair of adjacent edges have distinct total degrees. Hence $\widetilde{G}$ is neighbourly edge total irregular fuzzy graph.

Remark 3.3 Converse of the above two Theorems need not be true.

ftbpFU5.8297in0.9279in0ptCounter example14Figure(14)

Example 3.13 Consider $\widetilde{G}=(\sigma, \mu)$ be a fuzzy graph such that $G=(V, E)$ is a path on four vertices. From Figure $(14)$,

$d_{\widetilde{G}}(u)=0.6, d_{\widetilde{G}}(v)=1.2, d_{\widetilde{G}}(w)=1.2, d_{\widetilde{G}}(x)=0.6, d_{\widetilde{G}}(u, v)=0.6, d_{\widetilde{G}}(v, w)=1.2, d_{\widetilde{G}}(w, x)=0.6$. Here, $d_{\widetilde{G}}(u, v) \neq d_{\widetilde{G}}(v, w)$ and $d_{\widetilde{G}}(v, w) \neq d_{\widetilde{G}}(w, x)$. Hence $\widetilde{G}$ is neighbourly edge irregular fuzzy graph. But $\widetilde{G}$ is not strongly edge irregular fuzzy graph, since $d_{\widetilde{G}}(u, v)=d_{\widetilde{G}}(w, x)=0.6$. Also, $t d_{\widetilde{G}}(u, v)=1.2, t d_{\widetilde{G}}(v, w)=1.8, t d_{\widetilde{G}}(w, x)=1.2$. Note that $t d_{\widetilde{G}}(u, v) \neq t d_{\widetilde{G}}(v, w)$ and $t d_{\widetilde{G}}(v, w) \neq t d_{\widetilde{G}}(w, x)$. Hence $\widetilde{G}$ is neighbourly edge totally irregular fuzzy graph. But $\widetilde{G}$ is not strongly edge totally irregular fuzzy graph, since $t d_{\widetilde{G}}(u, v)=t d_{\widetilde{G}}(w, x)=1.2$.

Theorem 3.5 Let $\widetilde{G}=(\sigma, \mu)$ be a fuzzy graph on $G=(V, E)$, a star $K_{1, n}$. If the membership values of no two edges are the same, then $\widetilde{G}$ is strongly edge irregular fuzzy graph and $\widetilde{G}$ is totally edge regular fuzzy graph. 
Let $v_{1}, v_{2}, v_{3}, \ldots, v_{n}$ be the vertices adjacent to the vertex $x$.

Let $e_{1}, e_{2}, e_{3}, \ldots \ldots, e_{n}$ be the edges of a star $\widetilde{G}$ in that order having membership values $c_{1}, c_{2}, c_{3}, \ldots \ldots, c_{n}$ such that $c_{1} \neq c_{2} \neq c_{3}, \ldots \ldots, \neq c_{n}$. Then

$d_{\widetilde{G}}\left(e_{i}\right)=d_{\widetilde{G}}(x)+d_{\widetilde{G}}\left(v_{i}\right)-2 c_{i}=\left(c_{1}+c_{2}+c_{3}+\ldots \ldots+c_{n}\right)+c_{i}-2 c_{i},(1 \leq i \leq n)$.

$d_{\widetilde{G}}\left(e_{i}\right)=\left(c_{1}+c_{2}+c_{3}+\ldots \ldots+c_{n}\right)-c_{i},(1 \leq i \leq n)$.

All edges $e_{i},(1 \leq i \leq n)$ having distinct degrees.

Hence $\widetilde{G}$ is strongly edge irregular fuzzy graph.

Also, $t d_{\widetilde{G}}\left(e_{i}\right)=d_{\widetilde{G}}(x)+d_{\widetilde{G}}\left(v_{i}\right)-c_{i}$

$$
=\left(c_{1}+c_{2}+c_{3}+\ldots \ldots+c_{n}\right)+c_{i}-c_{i}, 1 \leq i \leq n, \text { and }
$$

$t d_{\widetilde{G}}\left(e_{i}\right)=\left(c_{1}+c_{2}+c_{3}+\ldots \ldots+c_{n}\right), 1 \leq i \leq n$.

All edges $e_{i}, 1 \leq i \leq n$, having same total degree.

Hence $\widetilde{G}$ is totally edge regular fuzzy graph.

Remark 3.4 A complete fuzzy graph need not be neighbourly irregular.

Example 3.14 Define $\widetilde{G}=(\sigma, \mu)$ by $\sigma(u)=0.5, \sigma(v)=0.4, \sigma(x)=0.7$,

$\sigma(w)=0.5$ and $\mu(u, v)=0.4, \mu(v, x)=0.4, \mu(x, w)=0.5$

$\mu(w, u)=0.5, \mu(u, x)=0.5, \mu(v, w)=0.4$,

$d_{\widetilde{G}}(u)=0.5+0.4+0.5+0.5=1.9, d_{\widetilde{G}}(v)=0.4+0.4+0.4+0.4=1.6, d_{\widetilde{G}}(x)=0.7+0.4+0.5+0.5=2.1$, $d_{\widetilde{G}}(w)=0.5+0.4+0.5+0.5=1.9$.

$\widetilde{G}$ is a complete fuzzy graph but not neighbourly irregular.

\section{Regular Fuzzy Graphs}

Definition 4.1 Let $\underset{G}{\widetilde{G}}=(\sigma, \mu)$ be a fuzzy graph on $G=(V, E)$. If $d_{\widetilde{G}}(v)=k$ for all $v \in V$, i.e. if each vertex has the same degree $k$, then $\widetilde{G}$ is said to be a regular fuzzy graph of degree $k$ or a $k$-regular fuzzy graph.

Example 4.1 Let $G=(V, E)$ where $V=\{u, v, w, x\}$ and $E=\{(u, v),(v, w),(w, x),(x, u)\}$, In Figure $(14)$, $d_{\widetilde{G}}(u)=$ $0.5, d_{\widetilde{G}}(v)=0.5, d_{\widetilde{G}}(w)=0.5, d_{\widetilde{G}}(x)=0.5$. Each vertex has the same degree 0.5 . Hence $\widetilde{G}$ is a regular fuzzy graphs of degree 0.5 .

ftbpFU3.4523in1.6924in0ptA regular fuzzy graph of degree 0.515Figure(15)

Definition 4.2 The total degree of a vertex $u$ is defined as $t d_{\widetilde{G}}(u)=\sum \mu(u v)+\sigma(u)=d_{\widetilde{G}}(u)+\sigma(u)$, $(u, v) \in E$. If each vertex of $\widetilde{G}$ has the same total degree $k$, then $\widetilde{G}$ is said to be the totally regular fuzzy graph of total degree $k$, or a k-totally regular fuzzy graph.

ftbpFU2.8236in1.8083in0ptRegular fuzzy graph and totally regular fuzzy graph.16Figure(16)

Example 4.2 Let $\widetilde{G}=(\sigma, \mu)$ be a fuzzy graph on $G=(V, E)$, an even cycle of length six. It is noted that in Figure

$(16), d_{\widetilde{G}}(u)=0.6, d_{\widetilde{G}}(v)=0.6, d_{\widetilde{G}}(w)=0.6, d_{\widetilde{G}}(x)=0.6, d_{\widetilde{G}}(y)=0.6, d_{\widetilde{G}}(z)=0.6 \Rightarrow \widetilde{G}$ is a $(0.6)-$ regular fuzzy graph. Also, note that $t d_{\widetilde{G}}(u)=1.0, t d_{\widetilde{G}}(v)=1.0, t d_{\widetilde{G}}(w)=1.0, t d_{\widetilde{G}}(x)=1.0, t d_{\widetilde{G}}(y)=1.0, t d_{\widetilde{G}}(x)=1.0$. 


\section{$4.1 d_{m}$-degree of a vertex in Fuzzy Graps}

Definition 4.3 Let $\widetilde{G}=(\sigma, \mu)$ be a fuzzy graph. The $d_{m}$-degree of a vertex $u$ in

$\widetilde{G}$ is $d_{m}(u)=\sum \mu^{m}(u, v)$, where

$\mu^{m}(u, v)=\sup \left\{\mu\left(u,, u_{1}\right) \wedge \mu\left(u_{1}, u_{2}\right) \wedge \ldots, \mu\left(u_{m-1}, v\right):\left(u, u_{1}, u_{2}, \ldots, u_{m-1}, v\right)\right.$ is

the shortest path connecting $u$ and $v$ of length $m$ \}.

Also, $\mu(u, v)=0$, for $(u, v)$ not in $E$.

The minimum $d_{m}$-degree of $\widetilde{G}$ is $\delta_{m}(\widetilde{G})=\wedge\left\{d_{m}(v): v \in V\right\}$.

The maximum $d_{m}$-degree of $\widetilde{G}$ is $\Delta_{m}(\widetilde{G})=\vee\left\{d_{m}(v): v \in V\right\}$.

Definition 4.4 Let $\widetilde{G}=(\sigma, \mu)$ be a fuzzy graph.

The $d_{2}$-degree of a vertex $u$ in $\widetilde{G}$ is $d_{2}(u)=\sum \mu^{2}(u, v)$, where

$\mu^{2}(u, v)=\sup \left\{\mu\left(u, u_{1}\right) \wedge \mu\left(u_{1}, v\right):\left(u, u_{1}, v\right)\right.$ is the shortest path

connecting $u$ and $v$ of length 2$\}$. Also, $\mu(u, v)=0$, for $(u, v)$ not in $E$.

The minimum $d_{2}$-degree of $\widetilde{G}$ is $\delta_{2}(G)=\wedge\left\{d_{2}(v): v \in V\right\}$.

The maximum $d_{2}$-degree of $\widetilde{G}$ is $\Delta_{2}(\widetilde{G})=\vee\left\{d_{2}(v): v \in V\right\}$.

Example 4.3 Consider $G=(V, E)$ where $V=\{u, v, w, x, y\}$ and

$E=\{(u, v),(v, w),(w, x),(x, y),(y, u)\}$. Define $\widetilde{G}=(\sigma, \mu)$

by $\sigma(u)=0.2, \sigma(v)=0.6, \sigma(w)=0.5, \sigma(x)=0.4, \sigma(y)=0.3$

and $\mu(u, v)=0.1, \mu(v, w)=0.3, \mu(w, x)=0.3, \mu(x, y)=0.2, \mu(y, u)=0.2 . f t b p F U 3.4523$ in2.4993in0ptMinimum $d_{2}$-degree of $\widetilde{G}$ and maximum17Figure(17)

In Figure $(17), d_{2}(u)=\{0.1 \wedge 0.3\}+\{0.2 \wedge 0.2\}=0.1+0.2=0.3$.

$d_{2}(v)=\{0.1 \wedge 0.2\}+\{0.3 \wedge 0.3\}=0.1+0.3=0.4$.

$d_{2}(w)=\{0.3 \wedge 0.2\}+\{0.3 \wedge 0.1\}=0.2+0.1=0.3$.

$d_{2}(x)=\{0.2 \wedge 0.2\}+\{0.3 \wedge 0.3\}=0.2+0.3=0.5$.

$d_{2}(y)=\{0.1 \wedge 0.2\}+\{0.2 \wedge 0.3\}=0.1+0.2=0.3$.

The minimum $d_{2}$-degree of $\widetilde{G}$ is

$\delta_{2}(G)=\wedge\left\{d_{2}(v): v \in V\right\}=\min \{0.3,0.4,0.3,0.5,0.3\}$

The maximum $d_{2}$-degree of $\widetilde{G}$ is

$\Delta_{2}(\widetilde{G})=\vee\left\{d_{2}(v): v \in V\right\}=\max \{0.3,0.4,0.3,0.5,0.3\}$

Definition 4.5 Let $\widetilde{G}=(\sigma, \mu)$ be a fuzzy graph on $G=(V, E)$. If $d_{m}(v)=k$ for all $v \in V$, then $\widetilde{G}$ is said to be $(m, k)$-regular fuzzy graph.

Definition 4.6 Let $\widetilde{G}=(\sigma, \mu)$ be a fuzzy graph on $G=(V, E)$. If $d_{2}(v)=k$ for all $v \in V$, then $G$ is said to be $(2, k)$-regular fuzzy graph.

Example 4.4 Consider $G=(V, E)$ where $V=\{u, v, w, x, y\}$ and $E=\{(u, v),(v, w),(w, x)\}$. 
Define $\widetilde{G}=(\sigma, \mu)$ by $\sigma(u)=0.2, \sigma(v)=0.3, \sigma(w)=0.4, \sigma(x)=0.5$, and $\mu(u, v)=0.2, \mu(v, w)=0.2, \mu(w, x)=0.2$.

In Figure $(18), d_{2}(u)=0.2, d_{2}(v)=0.2, d_{2}(w)=0.2, d_{2}(x)=0.2$.

This graph is $(2,0.2)$ - regular fuzzy graph.

ftbpFU2.1638in1.913in0pt(2,0.2)- regular fuzzy graph18Figure(18)

\subsection{Total $d_{m}$-degree of a vertex in Fuzzy Graph}

Definition 4.7 Let $\widetilde{G}=(\sigma, \mu)$ be a fuzzy graph on $G=(V, E)$. The total $d_{m}$-degree of a vertex $u \in V$ is defined as $t d_{m}(u)=\sum \mu^{m}(u, v)+\sigma(u)=d_{m}(u)+\sigma(u)$.

The minimum $t d_{m}$-degree of $\widetilde{G}$ is $t \delta_{m}(\widetilde{G})=\wedge\left\{t d_{m}(v): v \in V\right\}$.

The maximum $t d_{m}$-degree of $\widetilde{G}$ is $t \Delta_{m}(\widetilde{G})=\vee\left\{t d_{m}(v): v \in V\right\}$.

Now, total $d_{2}$-degree of a vertex in fuzzy graph is defined.

Definition 4.8 Let $\widetilde{G}=(\sigma, \mu)$ be a fuzzy graph on $G=(V, E)$.

The total $d_{2}$-degree of a vertex $u \in V$ is defined as

$t d_{2}(u)=\sum \mu^{2}(u, v)+\sigma(u)=d_{2}(u)+\sigma(u)$.

The minimum $t d_{2}$-degree of $\widetilde{G}$ is $t \delta_{2}(G)=\wedge\left\{t d_{2}(v): v \in V\right\}$.

The maximum $t d_{2}$-degree of $\widetilde{G}$ is $t \Delta_{2}(G)=\vee\left\{t d_{2}(v): v \in V\right\}$.

Example 4.5 Consider $G=(V, E)$ where $V=\{u, v, w, x\}$

and $E=\{(u, v),(v, w),(w, x),(x, u)\}$. Define $\widetilde{G}=(\sigma, \mu)$

by $\sigma(u)=0.2, \sigma(v)=0.3, \sigma(w)=0.4, \sigma(x)=0.5$.

and $\mu(u, v)=0.1, \mu(v, w)=0.3, \mu(w, x)=0.4, \mu(x, u)=0.2$.

In Figure (3.18), $d_{2}(u)=\operatorname{Sup}\{0.1 \wedge 0.3,0.2 \wedge .4\}=\operatorname{Sup}\{0.1,0.2\}=0.2$,

$d_{2}(v)=\operatorname{Sup}\{0.1 \wedge 0.2,0.3 \wedge 0.4\}=\operatorname{Sup}\{0.1,0.3\}=0.3$,

$d_{2}(w)=\operatorname{Sup}\{0.4 \wedge 0.2,0.3 \wedge 0.1\}=\operatorname{Sup}\{0.2,0.1\}=0.2$,

$d_{2}(x)=\operatorname{Sup}\{0.2 \wedge 0.1,0.4 \wedge 0.3\}=\operatorname{Sup}\{0.1,0.3\}=0.3$.

$t d_{2}(u)=0.2+0.2=0.4, t d_{2}(v)=0.3+0.3=0.6$,

$t d_{2}(w)=0.2+0.4=0.6, t d_{2}(x)=0.3+0.5=0.8$.

Definition 4.9 (Totally $(m, k)$-Regular Fuzzy Graph) If each vertex of $\widetilde{G}$ has the same total $d_{m}$ - degree $k$, then $\widetilde{G}$ is said to be totally $(m, k)$-regular fuzzy graph.

Definition 4.10 If each vertex of $\widetilde{G}$ has the same total $d_{2}$ - degree $k$, then $\widetilde{G}$ is said to be totally $(2, k)$-regular fuzzy graph.ftbpFU3.4731in2.5201in0ptA totally $(2, k)$-regular fuzzy graph need not be a $(2, k)$-regular fuzzy graph19Figure(19)

Example 4.6 1- A totally $(2, k)$-regular fuzzy graph need not be a $(2, k)$-regular fuzzy graph. Consider $G=(V, E)$ where $V=\{u, v, w, x, y\}$ and $E=\{(u, v),(v, w),(w, x),(x, y),(y, u)\}$. 
Note that in Figure (19),

$d_{2}(u)=0.6, d_{2}(v)=0.4, d_{2}(w)=0.5, d_{2}(x)=0.6, d_{2}(y)=0.5$ and

$t d_{2}(u)=1, t d_{2}(v)=1, t d_{2}(w)=1, t d_{2}(x)=1, t d_{2}(y)=1$.

Each vertex has same total $d_{2}$-degree 1 .

Hence $\widetilde{G}$ is totally $(2,1)$-regular fuzzy graph.

But $\widetilde{G}$ is not $(2, k)$-regular fuzzy graph.

2- A $(2, k)$-regular fuzzy graph need not be a totally $(2, k)$-regular fuzzy graph.

Consider $G=(V, E)$ where $V=\{u, v, w, x\}$ and $E=\{(u, v),(v, w),(w, x),(x, u)\}$.

In Figure $(20), d_{2}(u)=0.2, d_{2}(v)=0.2, d_{2}(w)=0.2, d_{2}(x)=0.2$

and $t d_{2}(u)=0.5, t d_{2}(v)=0.9, t d_{2}(w)=0.7, t d_{2}(x)=0.6$.

Each vertex has the same $d_{2}$-degree $(0.2)$.

So, $\widetilde{G}$ is $(2,0.2)$-regular fuzzy graph.

But $\widetilde{G}$ is not a totally $(2, k)$-regular fuzzy graph.

ftbpFU3.5993in1.5463in0ptA $(2, k)$-regular fuzzy graph need not be a totally $(2, k)$-regular fuzzy graph20Figure(20) 3- A $(2, k)$-regular fuzzy graph which is totally $(2, k)$-regular fuzzy graph.

Consider $G=(V, E)$ where $V=\{u, v, w, x, y, z\}$ and

$E=\{(u, v),(v, w),(w, x),(x, y),(y, z),(z, u)\}$.

In figure $(21), d_{2}(u)=0.6, d_{2}(v)=0.6, d_{2}(w)=0.6, d_{2}(x)=0.6$,

$d_{2}(y)=0.6, d_{2}(z)=0.6$ and $t d_{2}(u)=1, t d_{2}(v)=1, t d_{2}(w)=1$,

$t d_{2}(x)=1, t d_{2}(y)=1, t d_{2}(z)=1$.

Each vertex has the same $d_{2}$-degree (0.6).

So, $\widetilde{G}$ is a $(2,0.6)$-regular fuzzy graph.

Each vertex has the same total $d_{2}$-degree 1 .

Hence $\widetilde{G}$ is a totally $(2,1)$-regular fuzzy graph.

ftbpFU3.1808in1.9233in0ptA $(2, k)$-regular fuzzy graph which is totally $(2, k)$-regular fuzzy graph21Figure(21)

Theorem 4.1 Let $G=(\sigma, \mu)$ be fuzzy graph on $G=(V, E)$. Then $\sigma$ is constant function iff the following conditions are equivalent.

(i) $\widetilde{G}$ is $(2, k)$ - regular fuzzy graph.

(ii) $\widetilde{G}$ is totally $(2, k)$ - regular fuzzy graph.

Suppose that $\sigma$ is a constant function. Let $\sigma(u)=c$, constant for all $u \in V$.

Assume that $\widetilde{G}$ is $(2, k)$-regular fuzzy graph . Then $d_{2}(u)=k$, for all $u \in V$

So $t d_{2}(u)=d_{2}(u)+\sigma(u)$, for all $u \in V \Rightarrow t d_{2}(u)=k+c$, for all $u \in V$.

Hence $\widetilde{G}$ is totally $(2, k+c)$-regular fuzzy graph. Thus $(1) \Rightarrow(2)$ is proved.

Now suppose $\widetilde{G}$ is totally $(2, k)$ - regular fuzzy graph

$\Rightarrow t d_{2}(u)=k$, for all $u \in V$.

$\Rightarrow d_{2}(u)+\sigma(u)=k$, for all $u \in V$.

$\Rightarrow d_{2}(u)+c=k$, for all $u \in V$. 
$\Rightarrow d_{2}(u)=k-c$ for all $u \in V$.

Hence $\widetilde{G}$ is $(2, k-c)$ - regular fuzzy graph.

Hence $(i) \&(i i)$ are equivalent.

Conversely assume that $(i)$ and $(i i)$ are equivalent.

Suppose $\sigma$ is not a constant function. Then $\sigma(u) \neq \sigma(w)$,

for at least one pair $u, w \in V$.

Let $\widetilde{G}$ be a $(2, k)$-regular fuzzy graph. Then $d_{2}(u)=d_{2}(w)=k$. So

$t d_{2}(u)=d_{2}(u)+\sigma(u)=k+\sigma(u)$ and $t d_{2}(w)=d_{2}(w)+\sigma(w)=k+\sigma(w)$.

Since $\sigma(u) \neq \sigma(w) \Rightarrow k+\sigma(u) \neq k+\sigma(w) \Rightarrow t d_{2}(u) \neq t d_{2}(w)$.

So $\widetilde{G}$ is not totally $(2, k)$ - regular fuzzy graph which is a contradiction to

our assumption.

Let $\widetilde{G}$ be a totally $(2, k)$-regular fuzzy graph. Then $t d_{2}(u)=t d_{2}(w)$

$\Rightarrow d_{2}(u)+\sigma(u)=d_{2}(w)+\sigma(w)$

$\Rightarrow d_{2}(u)-d_{2}(w)=\sigma(w)-\sigma(u) \neq 0$

$\Rightarrow d_{2}(u) \neq d_{2}(w)$. So $\widetilde{G}$ is not $(2, k)$ - regular fuzzy graph which is a contradiction to our assumption. Hence $\sigma$ is a constant function.

\section{References}

[1] A. Rosenfeld. : Fuzzy graphs, In: L. A. Zadeh, K. S. Fu, M. Shimura, EDs. .:Fuzzy Sets and Their Applications, Academic press (1975), pp. 77-95.

[2] A. Nagoor Gani and K. Radha .:The degree of a vertex in some fuzzy graphs ,International Journal of algorithms, computing and Mathematics, 2(3), (2009), pp. 107-116.

[3] A. Nagoor Gani and K. Radha. .: On Regular Fuzzy graphs, Journal of Physical Science, (12), (2008), pp. 33-40.

[4] A. Nagoor Gani and M. Basheer Ahmed. .: Order and Size in fuzzy graph, Bulletin of pure and applied Sciences, 22E(1), (2003), pp. 145-148.

[5] A. Nagoor Gani and S. R. Latha. .: On irregular fuzzy graphs, Appl. Math. Sci.6 (2012), pp. 33-44.

[6] H. J. Zimmermann. : Fuzzy set Theory And its Applications, Second edition. Kluwer Academic Puplishers, Bosten, Dordrecht, London, (1990).

[7] J. N. Mordeson and P. S. Nair. .: Fuzzy graphs and Fuzzy hypergraphs, Physica verlag, (2000).

[8] K. H. Rosen .: Discrete Mathematic and Its Applications, Seventh Edition, McGraw Hill companies, America ,New York , (2012).

[9] K. Radha and N. Kumaravel. .: Some Properties of edge regular fuzzy graphs, Jamal Acad. Res. J. (2014), pp. $121-127$.

[10] K. R. Bhutani, and A. Rosenfeld. .: Fuzzy end nodes in fuzzy graphs, Information Sciences, (2003), pp. 319-322.

[11] K. R. Sandeep Narayan and M. S. Sunitha .: Connectivity in a Fuzzy Graph and its Complement, Gen. Math. Notes, 9(1), (2012), pp. 38-43.

[12] M. Hussain. .: Fuzzy Relations. Belking Institute of Technology School of Engineering Department of Mathematics and Science, (2010). 
[13] N. R. Santhi Maheswari and C. Sekar. .: On (2, k )-regular fuzzy graphs, G.Venkataswamy Naidu College, Kovilpatti- 628502, India, (2010).

[14] N. R. Santhi Maheswair and C. Sekar. .: On neighbourly edge irregular fuzzy graphs, International Journal of Mathematical Archive 6 (10) (2015), pp. 224-231.

[15] N. R. Santhi Maheswari and C. Sekar .: On strongly edge irregular fuzzy graphs. Kragu Jevac Journal of Mathematics, Volume 40(1), (2016), pp. 125-135.

[16] O. T. Manjusha, M. S. Sunitha .: Connected domination in fuzzy graphs using strong arcs, Annals of Fuzzy mathematics and Informatics, volume x, (2015), pp. 1-xx.

[17] P. Bhattachara. .: Some Remarks on Fuzzy Graphs, Pattern Reconition Lett.6 (1987), pp. 297-302.

[18] S. N. Sivanandam, S. Sumathi and S. N. Deepa. .: Introduction to Fuzzy Logic using MATLAB. Springer-verlag Berlin Heidelberg, (2007).

[19] S. P. Nandhini and E. Nandhini. .: Strongly irregular fuzzy geaphs, International Journal of Algorithms, Computing and Mathematics 2 (3) (2009), pp. 107-116. 